\title{
Substance Abuse during Adulthood Subsequent to the Experience of Physical Abuse and Psychological Distress during Childhood
}

\author{
S Longman-Mills, W Abel, W De La Haye
}

\begin{abstract}
Objective: This study investigated if there was a significant relationship between physical abuse during childhood and experiencing psychological distress and substance abuse among university students. Methods: This cross-sectional study utilized a questionnaire to collect retrospective data from 382 university students (103 males and 279 females) about their substance use patterns, level of psychological distress and their exposure to physical abuse. The data were then analysed using bivariate statistics.

Results: Most (61.8\%) participants met the criteria for being physically abused, however, only $27.2 \%$ recognized the experience as abuse. Another $38.9 \%$ of the students reported moderate to severe psychological distress. There was a significant relationship between being physically abused and experiencing higher levels of psychological distress $(\mathrm{p}<0.001)$. Cannabis was the most frequently utilized illicit drug (10.3\%) while alcohol was the most frequently utilized licit drug (37.4\%). Drug abuse was found to be significantly associated with being physically abused during childhood ( $\mathrm{p}<$ 0.05).

Conclusion: Even though the results obtained are not generalizable, this study has provided important preliminary information, that experiencing physical abuse increases the likelihood of having higher levels of psychological distress and becoming a substance abuser during adulthood; thereby identifying an overlooked area to target anti-drug use interventions.
\end{abstract}

Keywords: Maltreatment, psychological distress, physical abuse, substance abuse

WIMJ Open 2015; 2 (1): 7

\section{INTRODUCTION}

'Substance abuse', according to the Diagnostic and Statistical Manual of Mental Disorders IV (DSM-IV TR), is the repeated use of a substance, over a year, with there being adverse legal, interpersonal, occupational and/or health related outcomes (1). Substance abuse is a significant concern in Jamaica as confirmed by the 2001 national survey that found $11.9 \%$ of respondents to have substance-related problems (2). Many demand and supply reduction strategies have been implemented in Jamaica to reduce substance abuse. These strategies have included educational campaigns in primary, secondary and tertiary institutions as well as communities and workplaces (3). There has also been the enactment and the enforcement of anti-drug laws to prevent drug production and drug trafficking. Despite these strategies, drug abuse is thought to be on the rise among adolescents and young adults (4). The risk factors for substance abuse are known to be biological (5), environmental (6) and psychosocial (7) in nature. Biological factors may

From: Department of Community Health and Psychiatry, The University of the West Indies, Kingston 7, Jamaica.

Correspondence: Dr S Longman-Mills, Department of Community Health and Psychiatry, The University of the West Indies, Kingston 7, Jamaica. E-mail: samanthalongman@gmail.com provide a genetic susceptibility to substance abuse, while environmental factors influence the availability of psychoactive substances. However, whether an individual uses or abuses a substance is believed to be highly psychosocial in nature (7), but many psychosocial risk factors are often overlooked. Experiencing physical abuse is an overlooked psychosocial risk factor that has been implicated in substance abuse (8).

The physical disciplining of children is entrenched within the Jamaican culture and is widely endorsed as an effective parenting strategy. Parents typically employ an authoritarian parenting style, with even the simplest disobedience resulting in the spanking of the child (9). This disciplining technique sometimes results in severe abuse (10). Consequently, the 2004 Jamaican Child Care and Protection Act stipulated that persons less than eighteen years old should be protected from abuse, harm or even the threat of harm. However, 6276 cases of physical abuse were reported to the Office of Children's Registry between the years 2007 and 2011 (11). This large number of cases is alarming as research within North America suggests that experiencing physical abuse is associated with adverse developmental outcomes, including an increased likelihood of becoming a substance abuser (12). 
Physical abuse may lead to substance abuse, as increased levels of psychological distress may arise after exposure to physical abuse (8), making self-medicating with a substance more likely. Yet, on the other hand, some researchers suggest that the impact of being physically abused is culturally dependent and therefore, in a culture where corporal punishment is acceptable, the adverse effects would be minimal (13). In light of these contradictory perspectives, this research investigated whether being exposed to physical abuse is associated with higher levels of psychological distress and also an increased likelihood of being a substance abuser, within a cultural context where physical disciplining is considered acceptable.

\section{SUBJECTS AND METHODS}

This study was a part of a larger research that investigated childhood maltreatment and substance use within Latin American and Caribbean countries (14, 15). This present segment utilized systematic sampling techniques to collect retrospective data from 382 (103 males and 279 females) Jamaican undergraduate university students. Students were required to be 18 years and older in order to participate and power analysis identified the minimum sample size to be 311 .

\section{Informed consent}

Ethical approval was obtained from the University Hospital of the West Indies/University of the West Indies/Faculty of Medical Sciences Ethics Committee. Each participating student also provided informed consent, prior to completing the questionnaire, by signing a consent form that declared the risks and benefits of the study.

\section{Instruments}

This research utilized a questionnaire consisting of the physical abuse scale from the Adverse Childhood Experiences questionnaire [ACE] (16), the Kessler Psychological Distress Scale [K10] (17) and selected questions from the Inter-American Drug Abuse Control Commission/Organization of American States (CICAD/OAS) drug use questionnaire.

Adverse Childhood Experiences Questionnaire: The ACE is a retrospective questionnaire that seeks to provide a measure of childhood maltreatment, namely neglect, sexual, emotional and physical abuse. This questionnaire has been used by many researchers in a variety of cultural settings and has demonstrated adequate reliability and validity (16). Only the physical abuse scale of this instrument, which consists of four items requiring either 'yes' or 'no' responses, was utilized for this study.

Kessler Psychological Distress Scale: The K10 (17) is a benchmark measure of psychological distress. The K10 is a self-report questionnaire that is used worldwide to screen for anxiety and depressive symptoms, which may indicate the presence of a psychological disorder. This measure consists of 10 items, with five possible Likert response choices. High scores on this measure suggest an increased likelihood of being diagnosed with a psychological disorder $(18,19)$.

CICAD/OAS drug use questionnaire: The students' drug use and abuse behaviours were assessed using questions from the CICAD/OAS drug use questionnaire. This questionnaire was previously used within the Caribbean and was found to demonstrate good levels of validity. Questions were selected to measure drug use and also drug abuse according to the DSM-IV definition (1).

\section{Data collection}

The questionnaire was pretested by using think-aloud interviews to ensure that each item was interpreted as intended. A table of random numbers was utilized to identify the participating faculties from the selected university. Every third student within the vicinity of the faculty office was solicited to participate. Upon completing their questionnaire, students were thanked for their participation and given a phone card. The relationship between experiencing physical abuse and psychological distress, as well as experiencing physical abuse and later drug abuse, were investigated using bivariate tests (Chi-square).

\section{RESULTS}

A total of 382 students participated, 27\% were males and $73 \%$ were females. The mean age was 21.27 years \pm 4.39 years, with most students $(88.5 \%)$ being between the ages 18 and 24 years. The majority of the students $(65.4 \%)$ reported a middle-class socio-economic background. Of note, $27.2 \%$ of the students believed they were physically abused, however, $61.8 \%$ actually met the criteria of being physically abused. Additionally, 30.9\% reported that they had witnessed the physical abuse of a loved one.

\section{Psychological distress}

The students' level of psychological distress can be seen in Table 1. Of those students who were physically abused, $38.9 \%$ reported moderate to severe psychological distress symptoms, and were therefore more likely to be diagnosed with a psychological disorder. Chi-squared analysis also revealed that experiencing physical abuse was significantly associated with higher levels of psychological distress during adulthood: $\chi^{2}(3, \mathrm{~N}=368)=19.757, p<0.001$.

Table 1: Reported levels of psychological distress

\begin{tabular}{lrr}
\hline Level of psychological distress & \multicolumn{2}{c}{ Total } \\
& $(\boldsymbol{f})$ & $\mathbf{( \% )}$ \\
\hline Minimal & 153 & 41.6 \\
Mild & 93 & 25.3 \\
Moderate & 55 & 14.9 \\
Severe & 67 & 18.2 \\
\hline
\end{tabular}

\section{Psychoactive substance abuse}

Psychoactive substance use was reported by $38.9 \%$ of the students, with cannabis being the most frequently used illicit 
substance $(10.3 \%)$, while alcohol was the most frequently used licit drug [37.4\%] (Table 2). A non-significant relationship was identified between experiencing physical abuse and becoming a substance user: $\chi^{2}(1, \mathrm{~N}=380)=0.287, p=$ 0.592 .

Table 2: Reported frequency of substance use

\begin{tabular}{lrrrr}
\hline Substances & \multicolumn{2}{c}{ Past 12 months' use } & \multicolumn{2}{c}{ Past 30 days' use } \\
& $\boldsymbol{f}$ & $\mathbf{( \% )}$ & $\boldsymbol{f}$ & $\mathbf{( \% )}$ \\
\hline Cannabis & 39 & $(10.3)$ & 18 & $(4.7)$ \\
Tobacco/cigarettes & 34 & $(8.9)$ & 24 & $(6.3)$ \\
Alcohol & 142 & $(37.4)$ & 92 & $(24.2)$ \\
Cocaine & 3 & $(0.8)$ & 3 & $(0.8)$ \\
\hline
\end{tabular}

The dysfunctional outcomes that occurred as a result of psychoactive substance use were analysed and $33.8 \%$ of the substance users $(13.6 \%$ of the total sample), also met the DSM-IV criteria for substance abuse. Chi-squared analyses identified a significant relationship between experiencing physical abuse and later substance abuse: $\chi^{2}(1, \mathrm{~N}=382)=$ $5.846, p<0.05$.

\section{DISCUSSION}

This research found that two in every three students were physically abused, while a third of the substance users were also substance abusers. This study confirmed the thesis that there is an increased likelihood of becoming a substance abuser when an individual has experienced physical abuse during childhood. Furthermore, being physically abused was asso-ciated with experiencing psychological distress during adulthood. These results are consistent with research from North America indicating that being from a culture where corporal punishment is deemed acceptable does not act as a buffer against the adverse developmental outcomes associated with being physically abused. Notably, the majority of the students who were physically abused did not recognize the experience as abuse and yet they did experience higher levels of psychological distress and were more likely to become substance abusers.

Experiencing physical abuse was not found to be associated with substance use, but physically abused participants were significantly more likely to abuse a substance. These findings suggest that being physically abused is a risk factor for psychological distress and the abuse of psychoactive substances, but does not influence the use of a substance (12). This is a cause for concern as the majority of the participants were physically abused.

Psychological distress is more likely to occur after experiencing physical abuse, as being physically abused often results in emotion regulation difficulties and the utilization of avoidance coping strategies (8). Avoidance coping is an ineffective coping strategy as it involves denying, delaying, minimizing or suppressing stressful thoughts or emotions, which only amplifies the effect of the stressor.
Using this coping strategy makes one less likely to obtain social support (20) and is also associated with substance abuse.

Psychoactive substance abuse has persisted as a serious concern within Jamaica despite the numerous educational campaigns, rehabilitative treatments and anti-drug demand strategies that have been implemented. This suggests that there are other factors driving drug abuse in Jamaica that are not being targeted. One apparently overlooked area is the harsh physical disciplining that is often used by parents to facilitate obedience. Physical abuse has now been highlighted as being a risk factor for later substance abuse within the Jamaican context; thereby identifying a potential area to focus demand reduction strategies. Interventions aimed at preventing physical abuse and also enhancing cultural awareness of its potential adverse outcomes should be seen as an integral part of any drug demand reduction strategy for Jamaica.

\section{Limitations}

This was a self-report, retrospective study, with the sample being limited to young adult university students. Therefore, the results obtained cannot be generalized to the entire Jamaican population. Additionally, the responses obtained were vulnerable to the effects of memory interference and decay. Participants may have forgotten important information relating to their childhood experiences or may have repressed these memories. It should also be noted that in sensitive matters relating to abuse, there might be a tendency to under-report or over-report child abuse and drug use problems. Furthermore, social desirability may have influenced the responses given by the participants.

\section{ACKNOWLEDGEMENTS}

This study was financially sponsored by the Government of Canada/DFAIT, Organization of America States (OAS), Inter-American Drug Control Commission (CICAD) and the Centre for Addiction and Mental Health (CAMH)Toronto/Canada. However, the opinions expressed in this article are the sole and exclusive responsibility of the authors and do not represent the opinions of the supporting organizations or the organizations in which they are employed.

\section{REFERENCES}

1. American Psychiatric Association. Diagnostic and statistical manual of mental disorders. $4^{\text {th }}$ ed, text revision. Washington, DC: American Psychiatric Association; 2000.

2. National Council on Drug Abuse. National household survey of drug use and abuse in Jamaica, 2001. Kingston: NCDA; 2002.

3. Organization of American States. Jamaica. Evaluation of progress in drug control 2005-2006. Washington, DC: Organization of American States; 2006.

4. National Council on Drug Abuse. National School Survey 2006. Washington, DC: Organization of American States/Inter-American Drug Abuse Control Commission (OAS/CICAD); 2006. 
5. Kendler KS, Chen X, Dick D, Maes H, Gillespie N, Neale MC et al Recent advances in the genetic epidemiology and molecular genetics of substance use disorders. Nat Neurosci 2012; 15: 181-9. doi: 10.1038/nn.3018.

6. Boardman J, Finch B, Ellison C, Williams D, Jackson J. Neighborhood disadvantage, stress, drug use among adults. J Health Soc Behav 2001; 42: $151-65$.

7. Brook JS, Saar NS, Zhang C, Brook DW. Psychosocial antecedent and adverse health consequences related to substance use. Am J Public Health 2009; 99: 563-8.

8. Min M, Farkas K, Minnes S, Singer LT. Impact of childhood abuse and neglect on substance abuse and psychological distress in adulthood. J Trauma Stress 2007; 20: 833-44.

9. Smith DE, Mosby G. Jamaican child-rearing practices: the role of corporal punishment. Adolescence 2003; 38: 369-81.

10. Rodriguez CM. Parent-child aggression: association with child abuse potential and parenting styles. Violence Vict 2010; 25: 728-41.

11. Office of the Children's Registry. Statistics: physical abuse. Kingston, Jamaica: OCR; 2012 [cited 2012 July 30]; Available from: http://www. ocr.gov.jm/index.php/statistics/physical-abuse

12. Lo CC, Cheng TC. The impact of childhood maltreatment on young adults' substance abuse. Am J Drug Alcohol Abuse 2007; 33: 139-46.

13. Brown J, Johnson S. Childrearing and child participation in Jamaican families. Int J Early Years Educ 2008; 16: 31-40.

14. Longman-Mills S, González Y, Meléndez M, García M, Gómez J, Juárez $\mathrm{C}$ et al. Child maltreatment and its relationship to drug use in Latin America and the Caribbean: an overview and multinational research partnership. Int J Mental Health Addict 2011; 9: 347-64.

15. Longman-Mills S, González WY, Meléndez MO, García MR, Gómez JD, Juárez CG et al. Exploring child maltreatment and its relationship to alcohol and cannabis use in selected Latin American and Caribbean countries. Child Abuse Negl 2013; 37: 77-85. doi: 10.1016/j.chiabu. 2012.11.002. Epub 2013 Jan 7.
16. Centers for Disease Control and Prevention. Adverse Childhood Experiences (ACE) Study. Atlanta, GA: Centers for Disease Control and Prevention; 2012 [updated 2011 Jun 1; cited 2012 Aug 22]; Available from: www.cdc.gov/ace/questionnaires.htm

17. Kessler R, Barker P, Colpe L, Epstein J, Gfroerer J, Hiripi E et al. Screening for serious mental illness in the general population. Arch Gen Psychiatry $2003 ; 60: 184-9$.

18. Andrews G, Slade T. Interpreting scores on the Kessler Psychological Distress Scale (K10). Aust N Z J Public Health 2001; 25: 494-7.

19. Pratt L, Dey AN, Cohen AJ. Characteristics of adults with serious psychological distress as measured by the K6 Scale: United States 2001-04. Adv Data 2007; (382): 1-18.

20. Rudnicki SR, Graham JL, Habboushe DF, Ross RD. Social support and avoidant coping: correlates of depressed mood during pregnancy in minority women. Women Health 2001; 34: 19-34.

Submitted 13 Oct 2014

Accepted 27 Oct 2014

Published 16 Mar 2015

Online: http://www.mona.uwi.edu/wimjopen/article/1626

(C) Longman-Mills et al 2015

This is an open access article made freely available under Creative Commons Attribution 4.0 International (CC BY 4.0). Users are free to share, copy and adapt this work as long as the copyright holder (author) is appropriately and correctly credited. See http:// creativecommons.org/licences/by/4.0/deed.en_us for more information. 